\title{
Dispersive Two-Loop Calculations: Methodology and Applications
}

\section{A. Aleksejevs*}

Memorial University of Newfoundland, Grenfell Campus, Corner Brook, Canada

E-mail: aaleksejevs@grenfell.mun.ca

\section{S. Barkanova}

Memorial University of Newfoundland, Grenfell Campus, Corner Brook, Canada

E-mail: sbarkanova@grenfell.mun.ca

\begin{abstract}
As the new-generation precision experiments such as MOLLER [1] and P2 [2] look for physics beyond Standard Model, it is becoming increasingly important to evaluate the higher-order electroweak radiative corrections to a sub-percent level of uncertainty. However, due to propagators with different masses and higher-order tensor Feynman integrals, the two-loop calculations involving thousands of Feynman graphs become a demanding task requiring novel computational approaches. In this paper, we describe our dispersive sub-loop insertion approach and develop two-loop integrals using two-point functions basis which is applicable to wide range of processes.
\end{abstract}

XXIX International Symposium on Lepton Photon Interactions at High Energies - LeptonPhoton2019 August 5-10, 2019

Toronto, Canada

${ }^{*}$ Speaker. 


\section{Introduction}

In the past decade, the search for physics beyond the Standard Model (BSM) became one of the most important objectives in particle physics. The searches for BSM physics involve highenergy colliders, underground, ground and space telescopes, and high-precision experiments with high intensity beams at low energies. With high-precision searches, the measured observables, such as left-right (LR) or forward-backward (FB) parity-violating asymmetries, are extracted with uncertainties reaching a percent level. Any significant deviation between theoretical prediction based on the Standard Model (SM) calculation and experiment would be a definitive signal for BSM physics. The MOLLER experiment planned at JLab [1] is proposing to measure the PV asymmetry in the electron-electron scattering with the fractional accuracy of $2.4 \%$, which is more than a factor of five improvement over the precision of its predecessor experiment E-158 at SLAC [3]. The P2 experiment [2] proposes to measure PV asymmetry in electron-proton scattering with overall fractional accuracy at $1.4 \%$. Obviously, the theoretical uncertainty must be lower or at least match the experimental accuracy to make any conclusions regarding the BSM physics signal. The theoretical accuracy is mainly derived from propagation of uncertainty in input parameters and from limited knowledge of the higher order, i.e. beyond the one-loop level, contributions. Specifically, for the MOLLER experiment, the major electroweak two-loop corrections to the Born asymmetry evaluated in [4-8] in the on-shell renormalization scheme, were found to be close to five percent, which is a significant contribution compared to the expected experimental precision. Clearly, it is imperative to calculate a full set of two-loop diagrams participating in $e-e$ or $e-$ $p$ PV scattering, but this is not a straightforward task, and it will most probably require a high degree of automatization due to a very large number of diagrams. There is an extensive body of literature dedicated to the development of two-loop calculations [9-23], offering a wide spectrum of approaches. We have outlined our general approach to calculations of the two-loop diagrams based on the representation of many-point Passarino-Veltman (PV) functions in two-point function basis in [24-26]. Here, we were able to replace a sub-loop integral by the dispersive and regularized representation of the two-point function. As a consequence, the second-loop integral received an additional propagator and we were able to use the PV basis for the second-loop integration in the final stage of the calculations. In this paper, we outline some of the results obtained with the approach developed in [24-26].

\section{Sub-Loop Insertion}

We derive the main ideas in the dispersive sub-loop approach from the example of the selfenergy and triangle insertions. For the the left graph in Fig. 1, we can write the following two-loop integral:

$$
M_{a}=-\frac{2}{\pi^{4}} \int \frac{d^{4} q_{1} d^{4} q_{2}}{\left[q_{2}^{2}-m^{2}\right]\left[\left(q_{1}-q_{2}\right)^{2}-m^{2}\right]\left[q_{1}^{2}-m^{2}\right]^{2}\left[\left(k-q_{1}\right)^{2}-m^{2}\right]} .
$$

Here, we assume that all propagators represent scalar particles, couplings are set to one, and masses are the same. Integration over sub-loop momentum $q_{2}$ will result in the simple two-point function: 

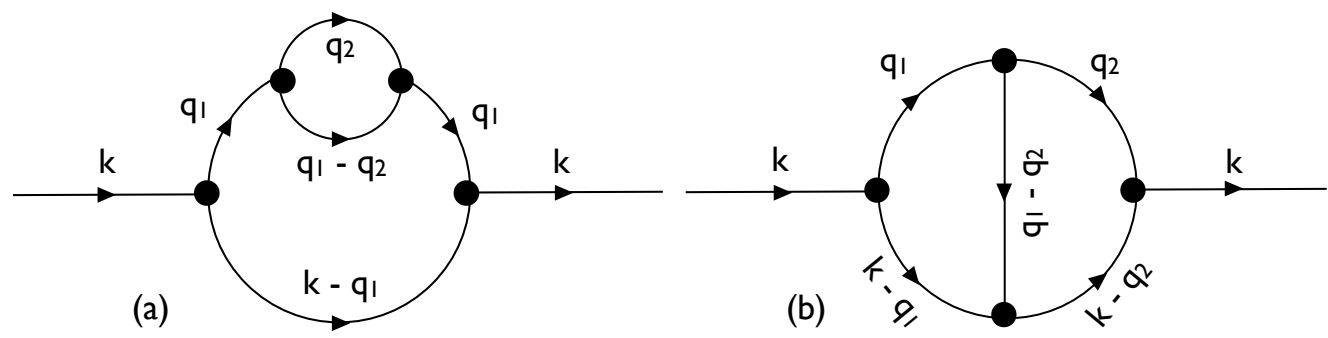

Figure 1: Two-loop self-energy and triangle insertions.

$$
\begin{aligned}
& B_{0}\left(q_{1}^{2}, m^{2}, m^{2}\right)=-\frac{i}{\pi^{2}} \int \frac{d^{4} q_{2}}{\left[q_{2}^{2}-m^{2}\right]\left[\left(q_{1}-q_{2}\right)^{2}-m^{2}\right]}: \\
& M_{a}=-\frac{2 i}{\pi^{2}} \lim _{\phi \rightarrow 0} \frac{\partial}{\partial \phi} \int \frac{B_{0}\left(q_{1}^{2}, m^{2}, m^{2}\right) d^{4} q_{1}}{\left[q_{1}^{2}-\left(m^{2}+\phi\right)\right]\left[\left(k-q_{1}\right)^{2}-m^{2}\right]} .
\end{aligned}
$$

To keep the results in the two-point function basis, we have removed quadratic form $1 /\left(q_{1}^{2}-m^{2}\right)^{2}$ and replaced it by $\lim _{\phi \rightarrow 0} \frac{\partial}{\partial \phi}\left(1 /\left[q_{1}^{2}-\left(m^{2}+\phi\right)\right]\right)$. Using [26], we can now represent dimensionally regularized two-point scalar function dispersively. Here, we also do not account for terms linear in $\varepsilon=\frac{4-D}{2}$, since in the Eq.2.2 $B_{0}$ function has $1 / \varepsilon$ pole but the second-loop integral is UV-finite:

$$
B_{0}\left(q_{1}^{2}, m^{2}, m^{2}\right)=\left(\frac{1}{\varepsilon}+\ln \frac{\mu^{2}}{m^{2}}\right)-\frac{q_{1}^{2}}{\pi} \int_{4 m^{2}}^{\infty} d s \frac{\mathfrak{J} B_{0}\left(s, m^{2}, m^{2}\right)}{s\left[q_{1}^{2}-s-i \omega\right]} .
$$

The last term in Eq.2.3 will contribute an additional propagator to the second loop, and using Eq.2.3 in Eq.2.2 we can produce the following two-loop result:

$$
\begin{aligned}
M_{a}= & \left.2\left(\frac{1}{\varepsilon}+\ln \frac{\mu^{2}}{m^{2}}\right) \partial_{\phi} B_{0}\left(k^{2}, m^{2}, m^{2}+\phi\right)\right|_{\phi=0} \\
& -\frac{2}{\pi} \int_{4 m^{2}}^{\infty} d s \frac{\Im B_{0}\left(s, m^{2}, m^{2}\right)}{s\left(s-m^{2}\right)^{2}}\left[\left.m^{2}\left(m^{2}-s\right) \partial_{\phi} B_{0}\left(k^{2}, m^{2}, m^{2}+\phi\right)\right|_{\phi=0}\right. \\
& \left.+s\left(B_{0}\left(k^{2}, m^{2}, s\right)-B_{0}\left(k^{2}, m^{2}, m^{2}\right)\right)\right] .
\end{aligned}
$$

Here, the two-point function and its derivative have the simple analytical structure:

$$
\begin{gathered}
B_{0}\left(k^{2}, m_{1}^{2}, m_{2}^{2}\right)=2+\left(\frac{1}{\varepsilon}+\ln \frac{\mu^{2}}{m_{2}^{2}}\right)+\frac{\sqrt{\lambda}}{k^{2}} \ln \frac{\left(\Delta_{+}+\sqrt{\lambda}\right)}{2 m_{1} m_{2}}-\frac{\Delta_{-}}{2 k^{2}} \ln \frac{m_{1}^{2}}{m_{2}^{2}}, \\
\left.\partial_{\phi} B_{0}\left(k^{2}, m^{2}, m^{2}+\phi\right)\right|_{\phi=0}=-\frac{1}{\sqrt{\Delta_{0} k^{2}}} \ln \frac{\sqrt{\Delta_{0} k^{2}}-k^{2}+2 m^{2}}{2 m^{2}},
\end{gathered}
$$


with $\Delta_{ \pm}=m_{1}^{2} \pm m_{2}^{2} \mp k^{2}, \Delta_{0}=k^{2}-4 m^{2}$, and $\lambda \equiv \lambda\left(k^{2}, m_{1}^{2}, m_{2}^{2}\right)=k^{4}+m_{1}^{4}+m_{2}^{4}-2\left(k^{2} m_{1}^{2}+k^{2} m_{2}^{2}+m_{1}^{2} m_{2}^{2}\right)$ is a usual Kallen function. In the final steps of calculations, the dispersive integration in the Eq.2.4 can be done numerically.

For the right graph in the Fig.1, we can write the two-loop integral as:

$$
M_{b}=-\frac{1}{\pi^{4}} \int \frac{d^{4} q_{1} d^{4} q_{2}}{\left[q_{2}^{2}-m^{2}\right]\left[\left(k-q_{2}\right)^{2}-m^{2}\right]\left[\left(q_{1}-q_{2}\right)^{2}-m^{2}\right]\left[q_{1}^{2}-m^{2}\right]\left[\left(k-q_{1}\right)^{2}-m^{2}\right]} .
$$

Clearly, the integration over momentum $q_{2}$ is represented by the three-point Passarino-Veltman function $C_{0}^{\{1\}} \equiv C_{0}\left(k^{2},\left(k-q_{1}\right)^{2}, q_{1}^{2}, m^{2}, m^{2}, m^{2}\right)=-\frac{i}{\pi^{2}} \int \frac{d^{4} q_{2}}{\left[q_{2}^{2}-m^{2}\right]\left[\left(k-q_{2}\right)^{2}-m^{2}\right]\left[\left(q_{1}-q_{2}\right)^{2}-m^{2}\right]}$. In order to replace $C_{0}^{\{1\}}$ function by the propagator-like structure, we need to write a dispersive representation of the three-point function. Using ideas from [24,26], we can use the Feynman trick to join the first two propagators in Eq.2.6, remove the quadratic form, and, after shifting momentum $q_{2}-q_{1}=\tau$, write $C_{0}^{\{1\}}$ function as:

$$
\begin{aligned}
C_{0}^{\{1\}} & =-\frac{i}{\pi^{2}} \lim _{\phi \rightarrow 0} \frac{\partial}{\partial \phi} \int_{0}^{1} d x \int \frac{d^{4} \tau}{\left[\tau^{2}-m^{2}\right]\left[\left(\tau-\left(k x-q_{1}\right)\right)^{2}-\left(m_{12}^{2}+\phi\right)\right]} \\
& =\lim _{\phi \rightarrow 0} \frac{\partial}{\partial \phi} \int_{0}^{1} d x B_{0}\left(\left(k x-q_{1}\right)^{2}, m^{2},\left(m_{12}^{2}+\phi\right)\right),
\end{aligned}
$$

where $m_{12}^{2}=m^{2}-k^{2} \bar{x} x$, and $\bar{x}$ is defined as $\bar{x}=1-x$. In the case when $k^{2}>4 m^{2}$, the mass parameter $m_{12}$ in the two-point function becomes imaginary for the values of $x \in\left(x_{1}, x_{2}\right)$, where $\left\{x_{1}, x_{2}\right\}$ are the real parts of roots of the equation $m^{2}-k^{2} \bar{x} x=0$. As a result, it is required to modify the dispersive representation of the two-point function. We provide the detailed discussion for the case of dispersive treatment of two-point functions with imaginary masses in [24]. Using [24] and [26], we can write the following:

$$
\begin{aligned}
& C_{0}^{\{1\}}=\int_{0}^{1} d x\left[\frac{1}{k^{2} \bar{x} x}\left(1+\frac{m^{2}}{k^{2} \bar{x} x} \ln \frac{m_{12}^{2}}{m^{2}}\right)+\left(k x-q_{1}\right)^{2} F\left(\left(k x-q_{1}\right)^{2}, m^{2}, m_{12}^{2}\right)\right], \\
& F\left(\left(k x-q_{1}\right)^{2}, m^{2}, m_{12}^{2}\right)= \begin{cases}\frac{1}{\pi} \int_{\left(m+m_{12}\right)^{2}}^{\infty} d s \frac{\mathfrak{S}\left[\left.\partial_{\phi} B_{0}\left(s, m^{2}, m_{12}^{2}+\phi\right)\right|_{\phi=0}\right]}{[s-i \omega]\left[s-\left(k x-q_{1}\right)^{2}-i \omega\right]}, & \text { if } x \in\left[0, x_{1}\right] \cup\left[x_{2}, 1\right] \\
\frac{1}{2 \pi i} \int_{-\infty}^{\infty} d s \frac{\left.\partial_{\phi} B_{0}\left(s, m^{2}, m_{12}^{2}+\phi\right)\right|_{\phi=0}}{[s-i \omega]\left[s-\left(k x-q_{1}\right)^{2}-i \omega\right]} & \text { if } x \in\left(x_{1}, x_{2}\right)\end{cases}
\end{aligned}
$$

and

$$
\left.\partial_{\phi} B_{0}\left(s, m^{2}, m_{12}^{2}+\phi\right)\right|_{\phi=0}=\frac{1}{2 s} \ln \frac{m^{2}}{m_{12}^{2}}-\frac{m^{2}-m_{12}^{2}+s}{s \sqrt{\lambda\left(s, m^{2}, m_{12}^{2}\right)}} \ln \frac{m^{2}+m_{12}^{2}-s+\sqrt{\lambda\left(s, m^{2}, m_{12}^{2}\right)}}{2 m m_{12}} .
$$


At this point, Eq.2.6 can be expressed using the three-point dispersive representation given in Eq.2.8:

$$
\begin{aligned}
M_{b}= & -\frac{i}{\pi^{2}} \int d^{4} q_{1} \frac{C_{0}^{\{1\}}}{\left[q_{1}^{2}-m^{2}\right]\left[\left(k-q_{1}\right)^{2}-m^{2}\right]} \\
= & \int_{0}^{1} \frac{d x}{k^{2} \bar{x} x}\left(1+\frac{m^{2}}{k^{2} \bar{x} x} \ln \frac{m_{12}^{2}}{m^{2}}\right) B_{0} \\
& -\frac{1}{\pi}\left(\int_{0}^{x_{1}} d x+\int_{x_{2}}^{1} d x\right)_{\left(m+m_{12}\right)^{2}}^{\infty} d s \frac{\mathfrak{I}\left[\left.\partial_{\phi} B_{0}\left(s, m^{2}, m_{12}^{2}+\phi\right)\right|_{\phi=0}\right]}{s-i \omega}\left(B_{0}^{\{1\}}+s C_{0}^{\{2\}}\right) \\
& -\frac{1}{2 \pi i} \int_{x_{1}}^{x_{2}} d x \int_{-\infty}^{\infty} d s \frac{\left.\partial_{\phi} B_{0}\left(s, m^{2}, m_{12}^{2}+\phi\right)\right|_{\phi=0}}{s-i \omega}\left(B_{0}^{\{1\}}+s C_{0}^{\{2\}}\right)
\end{aligned}
$$

where $B_{0}^{\{1\}} \equiv B_{0}\left(k^{2}, m^{2}, m^{2}\right)$ and $C_{0}^{\{2\}} \equiv C_{0}\left(k^{2}, k^{2} \bar{x}^{2}, k^{2} x^{2}, m^{2}, m^{2}, s\right)$. In Eq.2.9, the two-point function $B_{0}^{\{1\}}$ is UV divergent, but $M_{b}$ (right graph on Fig.1) is UV-finite and hence should not contain any dependence on $1 / \varepsilon^{n}$ or scale parameter $\mu$. This dependence cancels out when we calculate $M_{b}$ numerically, which provides a good test of Eq.2.9. For both dispersive and Feynman parameter
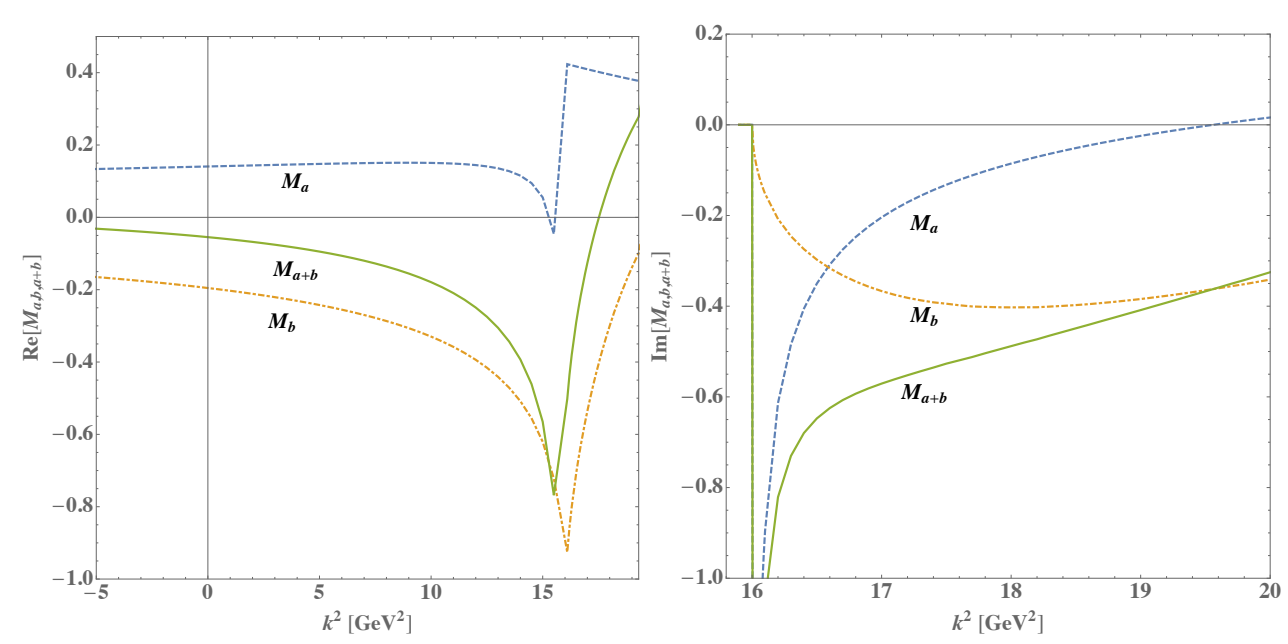

Figure 2: The left plot shows dependence of the real parts of Feynman diagrams shown on Fig.1 as a function of four-momentum squared $k^{2}$ for the $m=2.0(\mathrm{GeV})$. The right plot gives the dependence of imaginary parts of the same diagrams for the above threshold conditions $k^{2}>4 m^{2}$. For the UV regularization parameters $1 / \varepsilon$ and $\mu$, we use $1 / \varepsilon=0$ and $\mu=2.0(\mathrm{GeV})$.

numerical integration, we use Gauss-Kronrod integration library. In order to keep Feynman parameter integration stable, we have added a small imaginary part to the mass $m$. The results shown on 
Fig. 2 are given for both real and imaginary parts below and above the threshold conditions. On the left plot (see Fig.2), contributions from $\Re\left[M_{a}\right]$ and $\Re\left[M_{b}\right]$ into $\Re\left[M_{a+b}\right]$ do show some degree of cancellation in the space-like regime, and resonance type behaviour near the threshold. The computing time of the dispersion integral is in the order of fraction of a second. As for Feynman parameter integration, computing time highly depends on the threshold and is usually in the order of a few seconds per point below the threshold. Above the threshold, computing time raises dramatically (few minutes per point) due to the numerical noise at the points $x_{1}$ and $x_{2}$. Overall, Eq.2.4 and Eq.2.9, are in compact form and applicable for the broad kinematic region.

\section{Conclusion}

In this paper, we have outlined the dispersive treatment approach of the sub-loop insertion and represented the two-loop results in the two-point function basis. The second-loop integration was reduced to the one-loop type Feynman graph with an additional propagator coming from the dispersive sub-loop insertion. As an example, we chose the two-loop scalar self-energy calculations and the corresponding numerical results in Fig.2. The main conclusion of this work is that dispersive insertion approach simplifies analytical expressions considerably, to the point that it is possible to employ computer algebra evaluating the two-loop calculations analytically and carry out integration numerically.

\section{Acknowledgments}

The authors are grateful to the Lepton Photon 2019 organizing committee. This work is supported by the Natural Sciences and Engineering Research Council of Canada.

\section{References}

[1] MOLLER Collaboration (J. Benesch (Jefferson Lab) et al.), JLAB-PHY-14-1986 (2014) [nuclex/1411.4088].

[2] D. Becker (U. Mainz, PRISMA \& Mainz U., Inst. Kernphys.) et al., DOI: 10.1140/epja/i201812611-6 (2018) [nucl-ex/1802.04759].

[3] P. L. Anthony et al. [SLAC E158 Collaboration], Phys. Rev. Lett. 95, 081601 (2005) [arXiv:hep-ex/0504049].

[4] A. Aleksejevs, S. Barkanova, Y. Kolomensky, E. Kuraev, and V. Zykunov, Phys. Rev. D 85 (2012) 013007, arXiv:1110.1750.

[5] A. Aleksejevs, S. Barkanova, Y. M. Bystritskiy, E. A. Kuraev, A. N. Ilyichev, and V. Zykunov, (2012), arXiv:1202.0378.

[6] A. G. Aleksejevs, S. G. Barkanova, V. A. Zykunov and E. A. Kuraev, Phys. Atom. Nucl. 76 (2013) 888-900.

[7] A. Aleksejevs, S. Barkanova, Y. M. Bystritskiy, E. Kuraev, and V. Zykunov, Phys. Part. Nucl. Lett. 12, 645 (2015) arXiv:1504.03560.

[8] A. Aleksejevs, S. Barkanova, Yu. M. Bystritskiy, E. A. Kuraev, V. A. Zykunov, Phys. Part. Nucl. Lett. 13 (2016) no. 3, 310-317, arXiv:1508.07853. 
[9] D. Kreimer, Phys. Lett. B273 277-281 (1991).

[10] A. Czarnecki et al. Nucl. Phys. B433 259-275 (1995), [hep-ph/9405423].

[11] A. Frink et al. Nucl.Phys. B488 426-440 (1997), [hep-ph/9610285].

[12] L. Adams, C. Bogner, S. Weinzierl, J. Math. Phys. 54052303 (2013).

[13] L. Adams, C. Bogner, S. Weinzierl, J. Math. Phys. 56072303 (2015).

[14] L. Adams, C. Bogner, S. Weinzierl, J. Math. Phys. 57032304 (2016).

[15] E. Remiddi, L. Tancredi, Nucl.Phys. B907 400-444 (2016).

[16] S. Bloch, M. Kerr, P. Vanhove, Compos. Math. 151 2329-2375 (2015).

[17] S. Bloch, M. Kerr, P. Vanhove, Adv. Theor. Math. Phys. 21 1373-1453 (2017).

[18] S. Bauberger, M. Böhm, Nucl. Phys. B 445, 25-46 (1995).

[19] W. Hollik, U. Meier, S. Uccirati, Nucl. Phys. B731 213-224 (2005).

[20] A. Freitas, W. Hollik, W. Walter, G. Weiglein, Nucl. Phys. B632 189-218 (2002).

[21] M. Czakon, J. Gluza, T. Riemann, Phys.Rev. D71, 073009 (2005).

[22] S. Actis, M. Czakon, J, Gluza, and T. Riemannl., Phys. Rev. Lett. 100, 131602 (2008).

[23] M. Awramik, M. Czakon and A. Freitas, JHEP 0611048 (2006), [hep-ph/0608099].

[24] A. Aleksejevs, Phys. Rev. D 98, 036021 (2018) [hep-th/1804.08914].

[25] A. Aleksejevs (2018) [hep-th/1809.05592].

[26] A. Aleksejevs, S. Barkanova and A. Davydychev, (2019), [hep-th/1905.07936]. 